\title{
Elastic waves in particulate glass-rubber mixture: experimental and numeri- cal investigations/studies
}

\author{
Kianoosh Taghizadeh ${ }^{1, \star}$, Holger Steeb², Vanessa Magnanimo ${ }^{1}$, and Stefan Luding ${ }^{1}$ \\ ${ }^{1}$ Multi-Scale Mechanics, Faculty of Engineering Technology (ET), MESA+, University of Twente, Enschede, The Netherlands \\ ${ }^{2}$ Institute of Mechanics, University of Stuttgart, Stuttgart, Germany
}

\begin{abstract}
In this paper we study by wave propagation the elastic response of granular mixtures made of soft and stiff particles subjected under hydrostatic pressure/stress. This allows inferring fundamental properties of granular materials such as elastic moduli and dissipation mechanisms. We compare physical experiments in a triaxial cell equipped with piezoelectric wave transducers and Discrete Element Method simulations (DEM). In the experimental part, dense, static packings made of monodisperse glass and rubber beads are prepared at various levels of hydrostatic stress and species fractions. Small perturbations are generated on one side and the time of flight through the glass-rubber mixtures are measured to quantify the effect of the mixture composition on the elastic moduli. Interestingly, the experiments show that the behavior is non-linear and nonmonotonic with increasing percentage of rubber particles. Wave velocity and modulus remain fairly constant when increasing the fraction of rubber to $30 \%$, while they experience a sudden drop between $30 \%$ and $60 \%$, to become again constant between $60 \%$ to $100 \%$. DEM simulations offer deeper insights into the micromechanics in and at the transition between the glass- and rubber-dominated regimes. The simplest analysis with Hertzian spherical particles of different stiffness is performed as a preliminary step. The behavior of mixtures with high glass content is very well captured by the simulations, without need of any additional calibration, whereas the complex interaction between rubber and glass leave open questions for further study.
\end{abstract}

\section{Introduction}

In our daily life, we are surrounded by granular materials like soil, coffee, sand, nuts, etc. They constitute over $75 \%$ of raw materials feedstock to industry, including pharmaceutical, mining, agriculture, chemical, biotechnological, textile, etc. Despite its ubiquity, the behavior of granular materials is far from being fully understood, which leads to the loss of the world's energy consumption in processing and transport. Due to the wide range of application, particulate mixtures have received a lot of attention in the last decades. For example in geotechnical engineering recycled materials (e.g. shredded or granulated rubber, crushed glass) are often used into conventional designs and soil improvement projects [1-3]. Moreover, mixtures of asphalt and concrete are widely used to construct roads; thus exploring the effect of granular composition on the effective physical properties of mixtures can help optimizing industrial processes, engineering structures and make the pavement more robust and enduring [4-7].

The aim of this study is to explore the role of soft-stiff compositions on the bulk elastic response of the mixture. Particular attention is devoted to the dependence of sound velocity on the stress state, since this is an important controllable experimental parameter.

\section{Experiment}

\subsection{Experimental setup}

Uniform glass and rubber particles with similar size $\left(d_{r}\right.$ $=d_{g}=4 \mathrm{~mm}$ ) are used in this study to prepare cylindrical specimens with different volume fractions of glass and rubber. Material properties are reported in table 1. Let $v$ be the ratio between the volume of rubber particles and the total volume of solids in the mixture. Glass-rubber samples were prepared with variable rubber content, $v=0$, $0.05,0.1, \ldots, 0.9,1.0$, where $v=0$ composed of glass particles only and $v=1.0$ of rubber particles only. Care is taken to create homogeneous mixtures during manual inserting. Further segregation is avoided. All specimen are tested in our custom-made triaxial cell with sample diameter and height equal to $100 \mathrm{~mm}$. Fig. 1 shows a schematic drawing of the set up and peripheral electronics. Granular samples are compressed in the axial direction via the top piston in subsequent stress increments. At each instant step the radial stress is corrected to match the axial stress. Air is used as confining fluid for the samples enclosed by a rubber membrane. Seven pressure values are analyzed, namely $P=50,100,150,200,250,300$ and $350 \mathrm{kPa}$. At each pressure level, a high voltage burst signal is excited, to measure the time of flight. The top cap (the sound source) and the bottom plate (the detector) of the cell are instrumented with piezoelectric transducers with a diameter adjusted via a PMMT contact plate to the sample size $(100 \mathrm{~mm})$, able to excite longitudinal waves and measure

\footnotetext{
^e-mail: k.taghizadehbajgirani@utwente.nl
} 
Table 1. Properties of glass and rubber particles

\begin{tabular}{lll}
\hline Used material properties & Glass & Rubber \\
\hline Diameter $(\mathrm{mm})$ & 4 & 4 \\
Mass density $\left(\mathrm{kg} / \mathrm{m}^{3}\right)$ & 1540 & 860 \\
Shear modulus $(\mathrm{MPa})$ & 29000 & 200 \\
Poisson's ratio & 0.3 & 0.5 \\
\hline
\end{tabular}

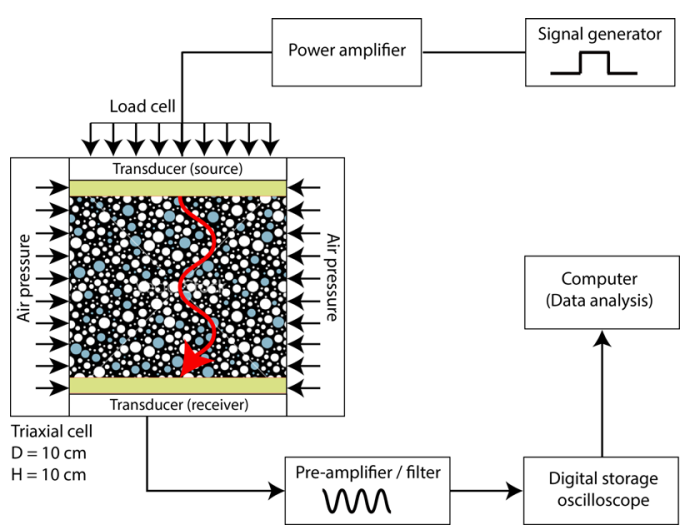

Figure 1. Schematic drawing of the experimental setup.

the arrival time, respectively. The transmitted signal is a $\pm 400 \mathrm{~V}$ step signal via the top cap. The signals transmitted and received are pre-amplified, filtered and recorded with a digital oscilloscope (LeCroy WaveSurfer 1GHz). Then, the wave velocity can be calculated from the travel time, given the height of the sample in the actual configuration and using the peak as reference. The signal-to-noise ratio is improved by repetitive averaging of 100 detected signals using the digital oscilloscope and then sent to a computer for further processing. We repeat the experiment five times for each rubber content and pressure level in order to avoid configuration-dependent results.

\subsection{Experimental observations}

In this section, we report results on the bulk stiffness of granular mixtures with diverse rubber content. In the longwavelength limit, the longitudinal, $\mathrm{P}$-wave modulus $M$ is related to the velocity $V_{p}$ in the medium by

$$
M=\rho V_{p}^{2},
$$

where $\rho$ is the mixture's density of the sample and is given by $\rho=(1-v) \rho_{g}+v \rho_{r}$, with $\rho_{g}$ and $\rho_{r}$ true mass densities of glass and rubber beads, respectively, and $v$ rubber content. By means of Eq. (2) we can then calculate the P-wave modulus of the granular mixtures tested in the triaxial cell. Fig. 2 shows the evolution of $M$ with rubber content, $v$ [\%], for all mixtures at different pressure levels. The figure shows that the compressional modulus remains fairly constant by increasing the volume of rubber content to $30 \%$. In the case of high pressure, adding a small amount of soft particles surprisingly enhances the effective stiffness of the medium and the highest modulus is observed at $v \approx$ $20 \%$. Hence, granular mixtures can be manipulated to obtain aggregates with even higher stiffness, but lighter and more dissipative thanks to rubber, when appropriate external conditions are matched (in this case the pressure) [8]. Between $30 \%$ and $60 \%$, there is a considerable drop in

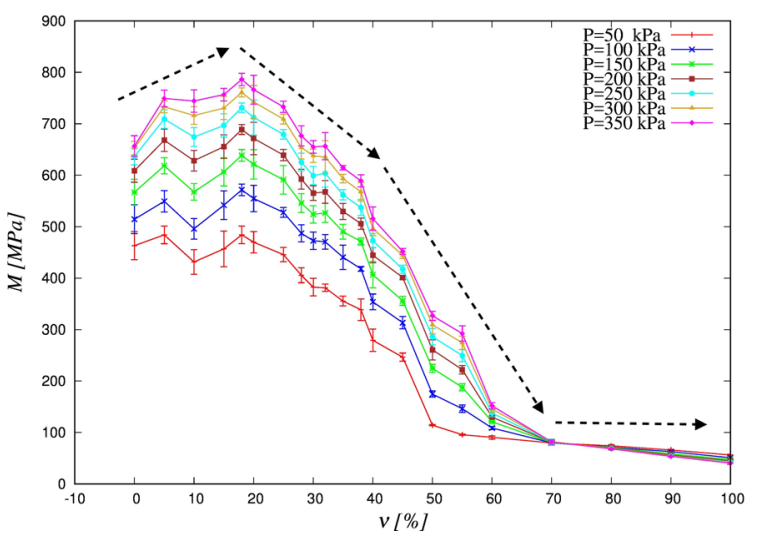

Figure 2. P-wave modulus versus fraction of rubber.

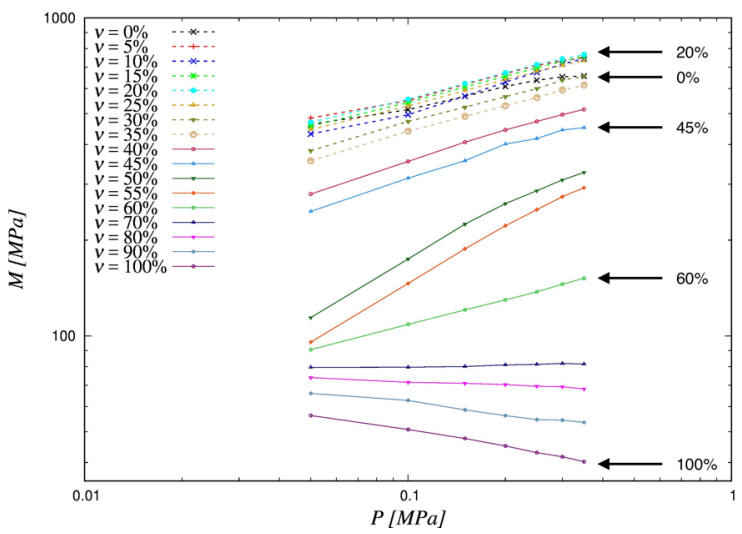

Figure 3. P-wave modulus versus applied vertical stress.

the wave velocity. The increased rubber-to-rubber interaction gradually reduces the effective stiffness as the rubber content increases. The modulus is again relatively stable between $60 \%$ to $100 \%$.

In Fig. 3, the same moduli are plotted against pressure $P$ in log-scale to evaluate their functional behaviour with $P$. The slopes of the $M$-lines with $P$ are almost constant for $v<50 \%$, while the behavior suddenly changes between $v=50 \%$ and $60 \%$ and the moduli become almost independent of pressure for higher rubber content. As already observed in Fig. 2, $70 \%$ to $100 \%$-rubber packings show little dependence on pressure and it is a surprise to see a little decrease in the moduli with increasing pressure.

\section{DEM study}

\subsection{Numerical setup}

To understand the experimental observations we perform numerical simulations and try to reproduce the behavior glass-rubber mixtures tested in the experimental part [9]. The Discrete Element Method (DEM) allows to simulate large number of interacting particles that either move following Newton's second law or just in static mechanical equilibrium (before the wave-tests) [10]. Given two elastic spheres $i$ and $j$ of radii $R_{i}$ and $R_{j}$ at position $\vec{x}_{i}$ and $\vec{x}_{j}$, the contact force in the normal direction is given by the Hertz contact law, by

$$
F_{n}=\frac{4}{3} E^{*} R^{1 / 2} \delta^{3 / 2}+F_{d}
$$


where $R=R_{i} R_{j} /\left(R_{i}+R_{j}\right)$ is the effective radius, $\delta=$ $\left[R_{i}+R_{j}-\left|\overrightarrow{x_{i}}-\overrightarrow{x_{j}}\right|\right]$ is the normal overlap and $E^{*}$ is the effective modulus

$$
\frac{1}{E^{*}}=\frac{1-v_{i}^{2}}{E_{i}}+\frac{1-v_{j}^{2}}{E_{j}},
$$

with $E_{i}, E_{j}$ and $v_{i}, v_{j}$ Young's moduli and Poisson's ratio, respectively. $F_{d}$ is the damping force between two particles and given by

$$
F_{d}=\gamma \sqrt{d \delta} \dot{\delta}
$$

where $\gamma$ is the Hertzian viscosity parameter and $\dot{\delta}$ is the relative velocity of two particles. Note that $F_{d}$ contributes very small value (almost zero) in case of quasi-static simulations since $\dot{\delta} \approx 0$ (particles do not move).

In a tangential direction a linear-dashpot contact model with Coulumb threshold is used with stiffness $k_{t}$ and damping $\gamma_{t}$ as defined in $[11,12]$ and with coefficient of particle friction $\mu=\mu_{s}=\mu_{d}$. To find the inter-species parameters (numerical value of parameter between a glass and a rubber particle), the reciprocals of parameters are added and the reciprocal of the sum is taken (product over sum) as the inter-species numerical value. For example, interspecies density between glass and rubber particles is given by $\rho_{g, r=\frac{\rho g}{\rho_{g}+\rho_{r}}}$, note that other contact properties follow the same rule. For glass and rubber particles the material characteristics in Table 1 and contact properties in Table 2 are used.

After defining the microscopic interaction between grains, we describe the protocol to generate packings and measure the elastic moduli. We start our simulations from a set of non-overlapping particles randomly generated in a periodical cubic box at an initial volume fraction $\phi=0.3$. The initial configuration is compressed isotropically by constant compression strain-rate until a given volume fraction $\phi=0.5$, below the jamming point. The system is then allowed to relax at constant volume fraction until it reaches a stable state, which means that the particles dissipate kinetic energy and achieve a zero-pressure unjammed relaxed configuration. This is followed by an isotropic compression until the desired maximum volume fraction, $\phi=0.82$ [13]. The same protocol is used for all glassrubber mixtures from $v=0.0$ to 1.0. In this way, samples resembling the set of experiments to some extent in Sec.2 are created. In simulations the preparation is isotropic, periodic without walls all the time, whereas in experiments, only hydrostatic stress conditions have been applied, thus isotropy of packing could not be guaranteed.

Once packings are created and compressed, various configurations are picked up at different pressure states above the jamming volume fraction. Those samples are allowed to relax with constant volume until a stable state is reached, which means the pressure remains unchanged over a period of time. Then a small strain perturbation is applied to these relaxed samples, either pure volumetric or pure deviatoric $[11,14]$. The bulk and shear moduli $B$ and $G$ are calculated as the ratio between the measured increment in stress and the applied strain:
Table 2. Summary and numerical values of particle parameters used in the DEM simulations [12]

\begin{tabular}{llll}
\hline Property & Symbol & Value & SI-units \\
\hline Time unit & $t$ & 1 & $10^{-6} \mathrm{~s}$ \\
Length unit & $x$ & 1 & $10^{-3} \mathrm{~m}$ \\
Mass unit & $m$ & 1 & $10^{-9} \mathrm{~kg}$ \\
Particle radius & $\langle a\rangle$ & 2 & $10^{-3} \mathrm{~m}$ \\
Number of particles & $N$ & 5000 & \\
Particle density, g & $\rho_{g}$ & 2540 & $2540 \mathrm{~kg} / \mathrm{m}^{3}$ \\
Particle density, $\mathrm{r}$ & $\rho_{r}$ & 1270 & $1270 \mathrm{~kg} / \mathrm{m}^{3}$ \\
Simulation time step & $\Delta t_{M D}$ & 0.01 & $10^{-8} \mathrm{~s}$ \\
Tangential stiffness, g & $k_{t}^{g}$ & $10^{4}$ & $10^{7} \mathrm{~kg} / \mathrm{s}$ \\
Tangential stiffness, $\mathrm{r}$ & $k_{t}^{r}$ & 150 & $150 \cdot 10^{3} \mathrm{~kg} / \mathrm{s}$ \\
Viscosity, g & $\gamma_{g}$ & 100 & $100 \mathrm{~kg} / \mathrm{s}$ \\
Viscosity, r & $\gamma_{r}$ & 100 & $100 \mathrm{~kg} / \mathrm{s}$ \\
Friction coefficient & $\mu$ & 0.5 & \\
\hline
\end{tabular}

$$
\begin{aligned}
B & =\frac{\delta P}{3 \delta \varepsilon_{v}}=\frac{\delta\left(\sigma_{x x}+\sigma_{y y}+\sigma_{z z}\right)}{3 \delta\left(\varepsilon_{x x}+\varepsilon_{y y}+\varepsilon_{z z}\right)}, \\
G_{x y} & =\frac{\delta\left(\sigma_{x x}-\sigma_{y y}\right)}{\delta\left(\varepsilon_{x x}-\varepsilon_{y y}\right)}, \text { with } \delta \varepsilon_{z z}=0,
\end{aligned}
$$

where $\sigma_{i j}$ and $\varepsilon_{i j}$ are components of stress and strain tensors respectively. Since the aggregates are isotropic, the $\mathrm{P}$-wave modulus is given by:

$$
M=B+\frac{4}{3} G
$$

as also measured directly by $M \simeq \frac{\delta \sigma_{x x}}{\delta \varepsilon_{x x}} \simeq \frac{\delta \sigma_{y y}}{\delta \varepsilon_{y y}} \simeq \frac{\delta \sigma_{z z}}{\delta \varepsilon_{z z}}$ when only one strain is activated.

\subsection{Numerical results}

In this section we show the results of the numerical moduli for different rubber content $v$ and pressure states. Note that a pressure range in simulation is wider than in experiments to gain more insight into the mechanical response of mixtures. In Fig.4 we plot the P-wave moduli versus pressure, decreasing monotonically with the rubber content from $v=0.0$ to $v=1.0$. In this respect, simulations are not able to reproduce the macroscopic behavior observed in Fig.3 in the experiment the maximum $M$ at $v \neq 0$. We associate the mismatch to the adopted contact model not appropriate to describe rubber-rubber and rubber-glass interactions. Finding a better contact model is subject to ongoing study. However, when experiments and simulations are directly compared in Fig.5, interesting information can be inferred. For the sake of clarity, only three cases are shown, namely, $v=0.05, v=0.5$ and $v=1.0$. Simulations with $v=0.05$ capture the experimental data quantitatively, noticeably without any calibration. On the other hand, when looking at the the packing $v=0.5$, the qualitative trend is well captured by simulations even if actual experimental values are higher than the simulated ones. Finally, for the case $v=1.0$, where the moduli are pressure independent, simulations are far from experiments in qualitative trend.

By summarizing the previous observations, a three regime 


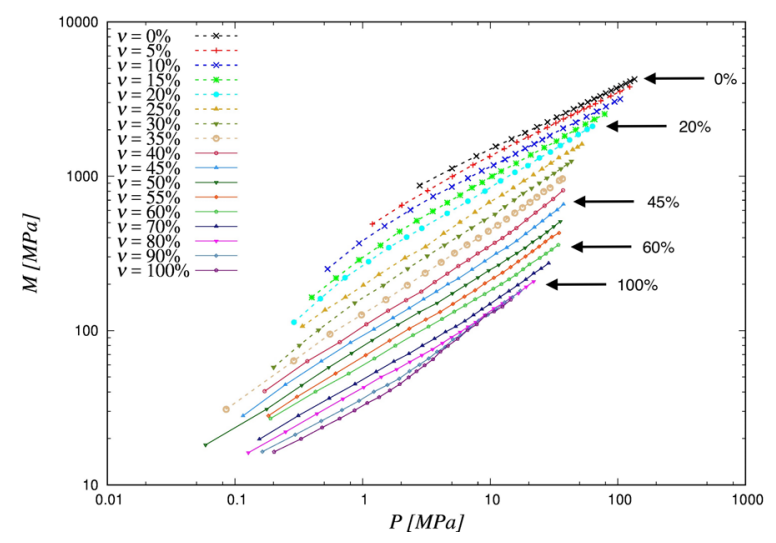

Figure 4. P-wave modulus versus applied vertical stress obtained by DEM simulations.

scenario shows up. In the glass-dominated regime $G$, waves do transmits via a glass beads network, where simulations based on Hertzian interactions are able to reproduce the macroscopic behavior. In the intermediate regime I, waves still have a preferential path via glass bead chains. Here two mechanisms concur to shape the bulk behavior: i) the density of glass beads in the sample reduces with respect to case $G$ and the actual values of the moduli get lower; ii) the number of contacts increases with pressure faster than in the G-regime due to easy rearrangement of the rubber particles, that is the slope $M(P)$ gets higher. Finally, in the third regime $\mathrm{R}$, the behavior of the mixture is dominated by the rubber beads, and the present simple DEM contact model can not offer an accurate representation of the system.

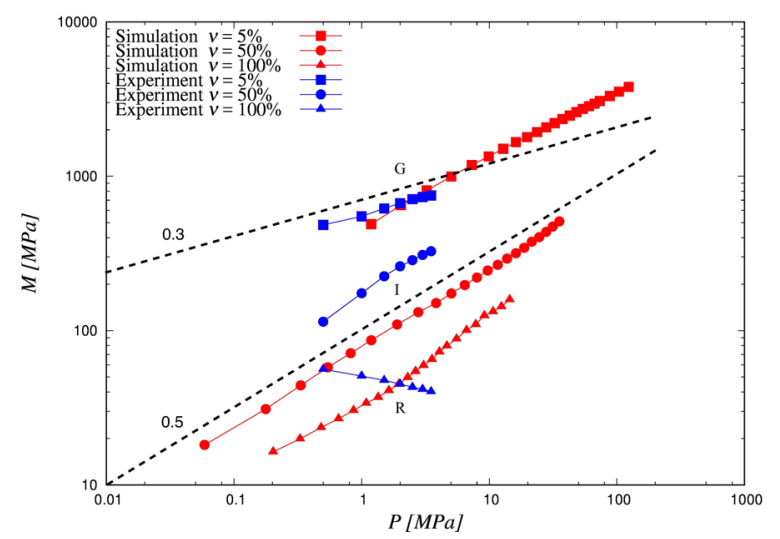

Figure 5. Experimental and numerical P-wave modulus plotted against pressure; comparison of DEM and experimental glassrubber mixtures for $v=0.05,0.5$ and 1.0 .

\section{Conclusion}

Experiments and numerical simulations were performed to examine the behavior of selected mixtures composed of glass and rubber particles. The experimental data indicate that the glass skeleton controls the behavior for $v<0.3$, while the rubber skeleton prevails at $v>0.6$. There is a considerable drop in modulus $M$ only at intermediate mixtures $(0.3<v<0.6)$ where the transition from stiff to soft occurs. Experimental data of high rubber content reveal that the modulus does not have any pressure dependency since there is not a strong stiff (glass) force chain for the wave to propagate through. Interestingly, we found that waves propagate faster in $v \approx 0.2$ than in for pure glass at all stress levels which might be of interest for many industrial applications to optimize their processes and materials.

Further, we studied numerically the evolution of granular mixtures with the volume fraction of rubber particles at different pressure levels to look more into the microstructure of the composites. Simulations data showed a systematical decrease of the P-wave modulus with increasing rubber fraction, unlike experimental observations where we could see a small peak of $M$ at $v \approx 0.2$. Comparing results between experiments and simulations, the noncalibrated numerical model captures well the experimental results for low rubber content samples and to some extent (for few cases of mixtures) also for really high $v$. However, simulations could not explain qualitatively the intermediate $v$ and the qualitative behavior of soft (rubber) dominated samples, because the Hertzian pair contact model is insufficient to depict the behavior of strongly deformable particles such as rubber. The classical contact laws predict the microstructure evolution during compression based on the assumption that contacts between particles are formulated locally as independent pair-interactions. In future, we thus will focus on the modification of contact models for strongly compressible particles [15].

\section{Acknowledgement}

The financial support of the European-Union Marie Curie Initial Training Network, T-MAPPP, funded by FP7 (ITN 607453), is appreciated, see http://www.t-mappp.eu/ for more information.

\section{References}

[1] P.J. Bosscher, et al, JGGE 123, 295 (1997)

[2] V.K. Garga, V. O'Shaughnessy, Canadian Geotechnical Journal 37, 75 (2000)

[3] J. Lee, et al, JGGE 125, 132 (1999)

[4] T.C. Heimdahl, A. Drescher, JGGE 125, 383 (1999)

[5] S. Pamukcu, S. Akbulut, JGGE 132, 501 (2006)

[6] J.G. Zornberg, et al, Canadian Geotechnical Journal 41, 227 (2004)

[7] B.H. Kjartanson, R.A. Lohnes, S. Yang (2002)

[8] H.K. Kim, J. Santamarina, Canadian Geotechnical Journal 45, 1457 (2008)

[9] T.M. Evans, J.R. Valdes, Granular Matter 13, 657 (2011)

[10] S. Luding, European Journal of Environmental and Civil Engineering 12, 785 (2008)

[11] K. Taghizadeh, et al, in IOP Conference Series: Earth and Environmental Science (IOP Publishing, 2015), Vol. 26, p. 012008

[12] S. Luding, Granular Matter 10, 235 (2008)

[13] N. Kumar, S. Luding, V. Magnanimo, Acta Mechanica 225, 2319 (2014)

[14] V. Magnanimo, et al, EPL (Europhysics Letters) 81, 34006 (2008)

[15] N. Brodu, J.A. Dijksman, R.P. Behringer, Physical Review E 91, 032201 (2015) 